\title{
A Note on Fuzzy Soft Topological Spaces
}

\author{
Vildan Çetkin ${ }^{1}$ Halis Aygün ${ }^{2}$ \\ ${ }^{1,2}$ Department of Mathematics, Kocaeli University, Umuttepe Campus, 41380, Kocaeli, TURKEY
}

\begin{abstract}
The main aim of this paper is to give a characterization of the category of fuzzy soft topological spaces and their continuous mappings, denoted by FSTOP. For this reason, we construct the category of antichain soft topological spaces and their continuous mappings, denoted by ASTOP. Also, we show that the category FSTOP is isomorphic to the category ASTOP.
\end{abstract}

Keywords: fuzzy soft set, fuzzy soft topology, soft I-topology.

\section{Introduction}

In 1999, Molodtsov [13] proposed a completely new concept called soft set theory to model uncertainty, which associates a set with a set of parameters. The soft set theory has been applied to many different fields with great success. Later, Maji et al. [11] introduced the concept of fuzzy soft set which combines fuzzy sets [17] and soft sets [13]. Soft set and fuzzy soft set theories have a rich potential for applications in several directions. So far, lots of spectacular and creative researches about the theories of soft set and fuzzy soft have been considered by some scholars (see $[3,7,8,9,12,15]$ ). Also, Aygünoğlu et al. [5] studied the topological structure of fuzzy soft sets based on the sense of Šstak [16].

The main result in this paper is that the category of compatible antichain soft topological spaces and their continuous mappings, denoted by ASTOP, is isomorphic to that of the category FSTOP of fuzzy soft topological spaces and their continuous mappings. This study is organized in the following manner. In the first and second sections, we give some fundamental concepts and notions about soft sets, fuzzy sets, fuzzy soft sets and fuzzy soft topology which are necessary for the last section. In the main section, we construct the category of antichain soft topological spaces and their continuous mappings, denoted by ASTOP, and show that the category FSTOP is isomorphic to the category ASTOP. Hence, we obtain the result that the category ASTOP is a topological category over $\mathbf{S E T}^{\mathbf{3}}$.

\section{Preliminaries}

Throughout this paper, $X$ refers to an initial universe, $E$ is the set of all parameters for $X, I=[0,1]$ and $I_{1}=I \backslash\{1\}$. For $\lambda \in[0,1], \bar{\lambda}(x)=\lambda$, for all $x \in X$.
Definition 2.1 [13] $F$ is called a soft set over $X$ if and only if $F$ is a mapping from $E$ into the set of all subsets of the set $X$, i.e., $F: E \rightarrow \mathcal{P}(X)$, where $\mathcal{P}(X)$ is the power set of $X$.

The value $F(e)$ is a set called $e$-element of the soft set for all $e \in E$. It is worth noting that the sets $F(e)$ may be arbitrary, empty, or have nonempty intersection. Thus a soft set over $X$ can be represented by the set of ordered pairs

$$
(F, E)=\{(e, F(e)) \mid e \in E\} .
$$

Example 2.1 (1) [7] Let $X=\left\{x_{1}, x_{2}, x_{3}, x_{4}, x_{5}\right\}$ be a universal set and $E=\left\{e_{1}, e_{2}, e_{3}, e_{4}\right\}$ be a set of parameters. If $F\left(e_{1}\right)=\left\{x_{2}, x_{4}\right\}, F\left(e_{2}\right)=$ $X, F\left(e_{3}\right)=F\left(e_{5}\right)=\emptyset$ and $F\left(e_{4}\right)=\left\{x_{2}, x_{3}, x_{5}\right\}$, then the soft set $F$ is written by

$$
(F, E)=\left\{\left(e_{1},\left\{x_{2}, x_{4}\right\}\right),\left(e_{2}, X\right),\left(e_{4},\left\{x_{1}, x_{3}, x_{5}\right\}\right)\right\} .
$$

(2) [14] For a topological space $(X, \tau)$, if $F(x)$ is the family of all open neighborhoods of a point $x \in X$, i.e., $F(x)=\{V \in \tau \mid x \in V\}$, then the ordered pair $(F, X)$ indeed a soft set over $\mathcal{P}(X)$.

Definition 2.2 [17] A fuzzy set on $X$ is a mapping $U: X \rightarrow I$, i.e., the family of all the fuzzy sets on $X$ is just $I^{X}$ consisting of all the mappings from $X$ to $I$. The value $U(x)$ represents the degree of $x$ belonging to the fuzzy set $U$.

A fuzzy set $U$ on $X$ can be represented as follows:

$$
U=\{(U(x) / x) \mid x \in X, U(x) \in I\}
$$

Definition 2.3 $[7,15] f$ is called a fuzzy soft set over $X$, where $f$ is a mapping from $E$ into $I^{X}$, i.e., $f_{e} \triangleq f(e): X \rightarrow I$ is a fuzzy set on $X$, for each $e \in E$.

Here, the value $f(e)$ is a fuzzy set called $e$-element of the fuzzy soft set for all $e \in E$. Thus, a fuzzy soft set $f$ over $X$ can be represented by the set of ordered pairs

$$
(f, E)=\left\{(e, f(e)) \mid e \in E, f(e) \in I^{X}\right\} .
$$

The family of all fuzzy soft sets over $X$ is denoted by $\left(I^{X}\right)^{E}$.

Example 2.2 [7] Let $X=\left\{x_{1}, x_{2}, x_{3}, x_{4}, x_{5}\right\}$ be a universal set and $E=\left\{e_{1}, e_{2}, e_{3}, e_{4}\right\}$ be a set of parameters. If $f\left(e_{1}\right)=\left\{0,9 / x_{2}, 0.5 / x_{4}\right\}, f\left(e_{2}\right)=$ $\overline{1}, f\left(e_{3}\right)=\overline{0}, f\left(e_{4}\right)=\left\{0.2 / x_{1}, 0.4 / x_{3}, 0.8 / x_{5}\right\}$, then the fuzzy soft set $f$ is written by

$$
\begin{aligned}
&(f, E)=\left\{\left(e_{1},\left\{0,9 / x_{2}, 0.5 / x_{4}\right\}\right),\left(e_{2}, \overline{1}\right),\left(e_{3}, \overline{0}\right),\right. \\
&\left.\left(e_{4},\left\{0.2 / x_{1}, 0.4 / x_{3}, 0.8 / x_{5}\right\}\right)\right\} .
\end{aligned}
$$

Definition $2.4[14,15]$ Let $f$ and $g$ be two fuzzy soft sets over $X$. Then 
(a) $f$ is called a fuzzy soft subset of $g$ and write $f \sqsubseteq g$ if $f_{e} \leq g_{e}$, for each $e \in E$. Two fuzzy soft sets $f$ and $g$ over $X$ are called equal if $f \sqsubseteq g$ and $g \sqsubseteq f$.

(b) the union of $f$ and $g$ is the fuzzy soft set $h=f \sqcup g$, where $h_{e}=f_{e} \vee g_{e}$, for each $e \in E$.

(c) the intersection of $f$ and $g$ is the fuzzy soft set $h=f \sqcap g$, where $h_{e}=f_{e} \wedge g_{e}$, for each $e \in E$.

Definition 2.5 The complement of a fuzzy soft set $f$ is denoted by $f^{\prime}$, where $f^{\prime}: E \longrightarrow I^{X}$ is a mapping given by $f_{e}^{\prime}=\overline{1}-f_{e}$, for each $e \in E$.

Clearly $\left(f^{\prime}\right)^{\prime}=f$.

Definition 2.6 [15] (Null fuzzy soft set) A fuzzy soft set $f$ over $X$ is called a null fuzzy soft set and denoted by $\Phi$, if $f_{e}=\overline{0}$, for each $e \in E$.

Definition 2.7 [15] (Absolute fuzzy soft set) A fuzzy soft set $f$ over $X$ is called an absolute fuzzy soft set and denoted by $\widetilde{E}$, if $f_{e}=\overline{1}$, for each $e \in E$. Clearly $(\widetilde{E})^{\prime}=\Phi$ and $\Phi^{\prime}=\widetilde{E}$.

Proposition 2.1 [2] Let $\Delta$ be an index set and $f, g, h, f_{i}, g_{i} \in\left(I^{X}\right)^{E}$, for all $i \in \Delta$. Then we have the following properties:

(1) $f \sqcap g=g \sqcap f, \quad f \sqcup g=g \sqcup f$.

(2) $f \sqcup(g \sqcup h)=(f \sqcup g) \sqcup h, f \sqcap(g \sqcap h)=(f \sqcap g) \sqcap h$.

(3) $f=f \sqcup(f \sqcap g), \quad f=f \sqcap(f \sqcup g)$.

(4) $f \sqcap\left(\bigsqcup_{i \in \Delta} g_{i}\right)=\bigsqcup_{i \in \Delta}\left(f \sqcap g_{i}\right)$.

(5) $f \sqcup\left(\sqcap_{i \in \Delta} g_{i}\right)=\sqcap_{i \in \Delta}\left(f \sqcup g_{i}\right)$.

Definition 2.8 [4] Let $\varphi: X_{1} \longrightarrow X_{2}$ and $\psi: E_{1} \longrightarrow E_{2}$ be two functions, where $E_{1}$ and $E_{2}$ are parameter sets for the crisp sets $X_{1}$ and $X_{2}$, respectively. Then the pair $\varphi_{\psi}$ is called a fuzzy soft mapping from $X_{1}$ to $X_{2}$.

Definition 2.9 [4] Let $f$ and $g$ be two fuzzy soft sets over $X_{1}$ and $X_{2}$, respectively and let $\varphi_{\psi}$ be a fuzzy soft mapping from $X_{1}$ to $X_{2}$.

(1) The image of $f$ under the fuzzy soft mapping $\varphi_{\psi}$, denoted by $\varphi_{\psi}(f)$, is the fuzzy soft set over $X_{2}$ defined by

$$
\varphi_{\psi}(f)_{k}(y)= \begin{cases}\bigvee_{\varphi(x)=y} \bigvee_{\psi(a)=k} f_{a}(x), & \text { if } x \in \varphi^{-1}(y) \\ 0, & \text { otherwise }\end{cases}
$$

for each $k \in E_{2}, y \in X_{2}$.

(2) The pre-image of $g$ under the fuzzy soft mapping $\varphi_{\psi}$, denoted by $\varphi_{\psi}^{-1}(g)$, is the fuzzy soft set over $X_{1}$ defined by $X_{1}$.

$\varphi_{\psi}^{-1}(g)_{e}(x)=g_{\psi(e)}(\varphi(x)), \quad$ for each $e \in E_{1}, x \in$

If $\varphi$ and $\psi$ are injective (surjective), then $\varphi_{\psi}$ is said to be injective (surjective).

(3) Let $\varphi_{\psi}$ be a fuzzy soft mapping from $X_{1}$ to $X_{2}$ and $\varphi_{\psi^{*}}^{*}$ be a fuzzy soft mapping from $X_{2}$ to $X_{3}$. Then the composition of these mappings from $X_{1}$ to $X_{3}$ is defined as follows: $\varphi_{\psi} \circ \varphi_{\psi^{*}}^{*}=\left(\varphi \circ \varphi^{*}\right)_{\psi \circ \psi^{*}}$, where $\psi: E_{1} \longrightarrow E_{2}$ and $\psi^{*}: E_{2} \longrightarrow E_{3}$.

Proposition 2.2 [9] Let $X_{1}$ and $X_{2}$ be two universes, $f, f_{1}, f_{2}, f_{i} \in\left(I^{X_{1}}\right)^{E_{1}}$ and $g, g_{1}, g_{2}, g_{i} \in$ $\left(I^{X_{2}}\right)^{E_{2}}$ for all $i \in \Delta$, where $\Delta$ is an index set. Then the following properties are satisfied:

(1) If $f_{1} \sqsubseteq f_{2}$, then $\varphi_{\psi}\left(f_{1}\right) \sqsubseteq \varphi_{\psi}\left(f_{2}\right)$.

(2) If $g_{1} \sqsubseteq g_{2}$, then $\varphi_{\psi}^{-1}\left(g_{1}\right) \sqsubseteq \varphi_{\psi}^{-1}\left(g_{2}\right)$.
(3) $\varphi_{\psi}\left(\bigsqcup_{i \in \Delta} f_{i}\right)=\bigsqcup_{i \in \Delta} \varphi_{\psi}\left(f_{i}\right)$.

(4) $\varphi_{\psi}\left(\prod_{i \in \Delta} f_{i}\right) \sqsubseteq \prod_{i \in \Delta} \varphi_{\psi}\left(f_{i}\right)$, the equality holds if $\varphi_{\psi}$ is injective.

(5) $\varphi_{\psi}^{-1}\left(\bigsqcup_{i \in \Delta} g_{i}\right)=\bigsqcup_{i \in \Delta} \varphi_{\psi}^{-1}\left(g_{i}\right)$.

(6) $\varphi_{\psi}^{-1}\left(\sqcap_{i \in \Delta} g_{i}\right)=\sqcap_{i \in \Delta} \varphi_{\psi}^{-1}\left(g_{i}\right)$.

\section{Fuzzy soft topological spaces}

To formulate our program and general ideas more precisely, first recall the concept of fuzzy topological space, that is of a pair $(X, \tau)$ where $X$ is a set and $\tau: I^{X} \rightarrow I$ is a mapping (satisfying some axioms) which assigns to every fuzzy set on $X$ the real number, which shows "to what extent" this set is open. According to this idea a fuzzy topology is a fuzzy set on $I^{X}$. This approach have lead us to define fuzzy soft topology which is compatible with the fuzzy soft theory. By our definition, a fuzzy soft topology is a fuzzy soft set over the set of all fuzzy soft sets $\left(I^{X}\right)^{E}$ which denotes "to what extent" the fuzzy soft set is open according to the parameters.

Throughout this study, let $E$ and $K$ be arbitrary nonempty sets viewed on the sets of parameters.

Definition 3.1 [5] A mapping $\tau: K \longrightarrow I^{\left(I^{X}\right)^{E}}$ is called a fuzzy $(E, K)$-soft topology on $X$ if it satisfies the following conditions for each $k \in K$ (where $\tau_{k} \triangleq \tau(k):\left(I^{X}\right)^{E} \rightarrow I$ is a mapping for each $k \in K)$ :

(O1) $\tau_{k}\left(\Phi_{X}\right)=\tau_{k}\left(\widetilde{E}_{X}\right)=1_{M}$.

(O2) $\tau_{k}(f \sqcap g) \geq \tau_{k}(f) \wedge \tau_{k}(g)$, for each $f, g \in$ $\left(I^{X}\right)^{E}$. $(\mathrm{O} 3) \tau_{k}\left(\bigsqcup_{i \in \Delta} f_{i}\right) \geq \bigwedge_{i \in \Delta} \tau_{k}\left(f_{i}\right)$, for each
$\left\{f_{i}\right\}_{i \in \Delta} \subseteq\left(I^{X}\right)^{E}$.

Then the pair $(X, \tau)$ is called a fuzzy $(E, K)$-soft topological space. The value $\tau_{k}(f)$ is interpreted as the degree of openness of a fuzzy soft set $f$ with respect to parameter $k \in K$.

Let $\tau^{1}$ and $\tau^{2}$ be two fuzzy $(E, K)$-soft topologies on $X$. We say that $\tau^{1}$ is finer than $\tau^{2}\left(\tau^{2}\right.$ is coarser than $\tau^{1}$ ), denoted by $\tau^{2} \leq \tau^{1}$, if $\tau_{k}^{2}(f) \leq \tau_{k}^{1}(f)$ for each $k \in K, f \in\left(I^{X}\right)^{E}$.

Example 3.1 [5] Let $\mathcal{T}$ be a fuzzy topology on $X$ in Šostak's sense, that is, $\mathcal{T}$ is a mapping from $I^{X}$ to $I$. Take $E=I$ and define $\overline{\mathcal{T}}: E \longrightarrow I^{X}$ as $\overline{\mathcal{T}}(e) \triangleq$ $\{\mu: \mathcal{T}(\mu) \geq e\}$ which is levelwise fuzzy topology of $\mathcal{T}$ in Chang's sense [6], for each $e \in I$. However, it is well known that each Chang's fuzzy topology can be considered as Šostak fuzzy topology. Hence, $\mathcal{T}(e)$ satisfies $(\mathrm{O} 1),(\mathrm{O} 2)$ and $(\mathrm{O} 3)$.

According to this definition and by using the decomposition theorem of fuzzy sets [10], if we know the resulting fuzzy soft topology, then we can find the first fuzzy topology. Therefore, we can say that a fuzzy topology can be uniquely represented as a fuzzy soft topology.

Example 3.2 Let $X=\{x, y\}, K=\left\{k_{1}, k_{2}\right\}$ and $E$ be a nonempty arbitrary parameter set for $X$. Define the mapping, $\tau:\left\{k_{1}, k_{2}\right\} \rightarrow I^{\left(I^{X}\right)^{E}}$ as follows: for each $e \in E$, 


$$
\begin{gathered}
\tau_{k_{1}}(f)= \begin{cases}\frac{1}{4}, & \text { if } f_{e}(x)>f_{e}(y) \\
\frac{1}{5}, & \text { if } f_{e}(x)<f_{e}(y) \\
1, & \text { if } f_{e}(x)=f_{e}(y)\end{cases} \\
\tau_{k_{2}}(f)= \begin{cases}\frac{9}{10}, & \text { if } f_{e}(x)>f_{e}(y) \\
\frac{8}{10}, & \text { if } f_{e}(x)<f_{e}(y) \\
1, & \text { if } f_{e}(x)=f_{e}(y)\end{cases}
\end{gathered}
$$

It is easy to verify that $\tau$ is a fuzzy $(E, K)$-soft topology on $X$.

Definition 3.2 [5] Let $\left(X_{1}, \tau^{1}\right)$ be a fuzzy $\left(E_{1}, K_{1}\right)$-soft topological space and $\left(X_{2}, \tau^{2}\right)$ be a fuzzy $\left(E_{2}, K_{2}\right)$-soft topological space. Let $\varphi$ : $X_{1} \longrightarrow X_{2}, \psi: E_{1} \longrightarrow E_{2}$ and $\eta: K_{1} \longrightarrow K_{2}$ be functions. Then the mapping $\varphi_{\psi, \eta}$ from $X_{1}$ into $X_{2}$ is called a fuzzy soft continuous map if $\tau_{k}^{1}\left(\varphi_{\psi}^{-1}(g)\right) \geq \tau_{\eta(k)}^{2}(g)$ for all $g \in\left(I^{X_{2}}\right)^{E_{2}}, k \in K_{1}$.

The category of fuzzy soft topological spaces and fuzzy soft continuous mappings is denoted by FSTOP.

Theorem 3.1 [5] The category FSTOP is a topological category over $\mathbf{S E T}^{3}$ with respect to the forgetful functor $V: \mathbf{F S T O P} \rightarrow \mathbf{S E T}^{3}$ which is defined by $V(X, \tau)=(X, E, K)$ and $V\left(\varphi_{\psi, \eta}\right)=$ $(\varphi, \psi, \eta)$.

\section{The category of ASTOP}

Definition 4.1 A parameterized family $\mathcal{T}=$ $\left\{\mathcal{T}_{k}\right\}_{k \in K}$ of $\mathcal{T}_{k} \subseteq\left(I^{X}\right)^{E}$ which satisfies the following properties for each $k \in K$ is called the $(E, K)$-soft $I$-topology on $X$.

(S1) $\Phi_{X}, \widetilde{E}_{X} \in \mathcal{T}_{k}$.

(S2) If $f, g \in \mathcal{T}_{k}$, then $f \sqcap g \in \mathcal{T}_{k}$.

(S3) If $\left\{f_{i}\right\}_{i \in \Gamma} \subseteq \mathcal{T}_{k}$, then $\bigsqcup_{i \in \Gamma} f_{i} \in \mathcal{T}_{k}$.

The pair $(X, \mathcal{T})$ is called an $(E, K)$-soft $I$ topological space.

Let $\left(X_{1}, \mathcal{T}^{1}\right)$ be an $\left(E_{1}, K_{1}\right)$-soft $I$-topological space, $\left(X_{2}, \mathcal{T}^{2}\right)$ be an $\left(E_{2}, K_{2}\right)$-soft $I$-topological space, $\varphi: X_{1} \rightarrow X_{2}, \psi: E_{1} \rightarrow E_{2}$ and $\eta: K_{1} \rightarrow K_{2}$ be functions. Then the mapping $\varphi_{\psi, \eta}:\left(X_{1}, \mathcal{T}^{1}\right) \rightarrow$ $\left(X_{2}, \mathcal{T}^{2}\right)$ is called continuous if

$$
g \in \mathcal{T}_{\eta(k)}^{2} \text { implies } \varphi_{\psi}^{-1}(g) \in \mathcal{T}_{k}^{1}, \text { for each } k \in K .
$$

Example 4.1 (1) The family $\mathcal{T}^{t}=\left\{\mathcal{T}_{k}^{t}\right\}$ is called trivial $(E, K)$-soft $I$-topology on $X$ where for each $k \in K, \mathcal{T}_{k}^{t}=\left\{\Phi_{X}, \widetilde{E}_{X}\right\}$.

(2) The family $\mathcal{T}^{D}=\left\{\mathcal{T}_{k}^{D}\right\}$ is called discrete $(E, K)$-soft $I$-topology on $X$ where for each $k \in K$, $\mathcal{T}_{k}^{D}=\left(I^{X}\right)^{E}$.

Given a set $X$, let $\mathbf{S T O}(X)$ denote all $(E, K)$-soft $I$-topologies on $X$. Consider the partial order $\subseteq$ on $\operatorname{STO}(X)$ which is defined as follows:

$$
\mathcal{T} \subseteq \mathcal{S}: \Leftrightarrow \mathcal{T}_{k} \subseteq \mathcal{S}_{k}, \text { for each } k \in K .
$$

Then, according to this order, the union and the intersection of two $(E, K)$-soft $I$-topologies
$\mathcal{T}=\left\{\mathcal{T}_{k}\right\}_{k \in K}$ and $\mathcal{S}=\left\{\mathcal{S}_{k}\right\}_{k \in K}$ on $X$ are described by the following equalities:

$\mathcal{T} \cup \mathcal{S}=\left\{(\mathcal{T} \cup \mathcal{S})_{k}\right\}_{k \in K}$ and $\mathcal{T} \cap \mathcal{S}=\left\{(\mathcal{T} \cap \mathcal{S})_{k}\right\}_{k \in K}$,

where $(\mathcal{T} \cup \mathcal{S})_{k}=\left\{\mathcal{T}_{k} \cup \mathcal{S}_{k}\right\}$ and $(\mathcal{T} \cap \mathcal{S})_{k}=$ $\left\{\mathcal{T}_{k} \cap \mathcal{S}_{k}\right\}$.

Hence $\mathbf{S T O}(X)$ is a complete lattice with respect to the order $\subseteq$ which is defined above, with the least element $\mathcal{T}^{t}$ and the greatest element $\mathcal{T}^{D}$.

Definition 4.2 An object of the category ASTOP is a pair $(X, \gamma)$, where $X$ is a set and $\gamma:[0,1] \rightarrow \mathbf{S T O}(X)$ is a map such that for each $a \in I_{1}, \gamma(a)=\bigcup_{b>a} \gamma(b)$ and $\gamma(1)=\left\{\Phi_{X}, \widetilde{E}_{X}\right\}$.

A morphism $\varphi_{\psi, \eta}:\left(X_{1}, \gamma^{1}\right) \rightarrow\left(X_{2}, \gamma^{2}\right)$ in ASTOP is a map $\varphi_{\psi, \eta}:\left(X_{1}, E_{1}, K_{1}\right) \rightarrow$ $\left(X_{2}, E_{2}, K_{2}\right)$ such that for each $a \in[0,1], \varphi_{\psi, \eta}$ : $\left(X_{1}, \gamma^{1}(a)\right) \rightarrow\left(X_{2}, \gamma^{2}(a)\right)$ is continuous.

An object $(X, \gamma)$ of ASTOP is called a compatible antichain $(E, K)$-soft $I$-topological space and $\gamma$ is said to be a compatible antichain $(E, K)$-soft $I$-topology on $X$.

Given a fuzzy $(E, K)$-soft topology $\tau$ on $X$, we can obtain a collection of $(E, K)$-soft $I$-topologies $\left\{\tau^{a} \mid a \in[0,1]\right\}$ on $X$, where $\tau_{k}^{a}=\left\{f \in\left(I^{X}\right)^{E} \mid\right.$ $\left.\tau_{k}(f) \geq a\right\}$. Moreover, if we let $\gamma_{k}^{\tau}(a)=\bigcup_{b>a} \tau_{k}^{b}$, then $\left(X, \gamma^{\tau}\right)$ is an object of ASTOP.

Lemma 4.1 Let $\tau$ be any fuzzy $(E, K)$-soft topology on $X$. Then for each $f \in \gamma_{k}^{\tau}(a), \tau_{k}(f) \geq a$.

Proof. It is trivial and therefore omitted.

Proposition 4.1 (1) If two fuzzy $(E, K)$-soft topologies on $X$ determine the same object in ASTOP, then they are equal.

$(2)$ Let $(X, \mathcal{S})$ and $(Y, \mathcal{T})$ be fuzzy $\left(E_{1}, K_{1}\right)$-soft topological space and fuzzy $\left(E_{2}, K_{2}\right)$-soft topological space, respectively. If $\varphi_{\psi, \eta}:(X, \mathcal{S}) \rightarrow(Y, \mathcal{T})$ is continuous, then $\varphi_{\psi, \eta}:\left(X, \gamma^{\mathcal{S}}\right) \rightarrow\left(Y, \gamma^{\mathcal{T}}\right)$ is continuous.

Proof. (1) Let $\mathcal{T}$ and $\mathcal{S}$ be two fuzzy $(E, K)$ soft topologies on $X$ satisfying $\gamma^{\mathcal{T}}=\gamma^{\mathcal{S}}$. We want to show $\mathcal{T}=\mathcal{S}$, i.e., for each $k \in K$ and $f \in$ $\left(I^{X}\right)^{E}, \mathcal{T}_{k}(f)=\mathcal{S}_{k}(f)$.

Let $k \in K$ and $a \in I_{1}=I \backslash\{1\}$ with $a<\mathcal{T}_{k}(f)$. Fix a number $b \in I_{1}$ such that $a<b \leq \mathcal{T}_{k}(f)$. Then $f \in \mathcal{T}_{k}^{b} \subseteq \gamma_{k}^{\mathcal{T}}(a)=\gamma_{k}^{\mathcal{S}}(a)$. By Lemma 4.1 , we get $\mathcal{S}_{k}(f) \geq a$. Therefore, since $a \in I_{1}$ is arbitrary, we obtain the following inequality $\mathcal{T}_{k}(f) \leq \mathcal{S}_{k}(f)$. On the other hand, by the similar way, we can show $\mathcal{S}_{k}(f) \leq \mathcal{T}_{k}(f)$. Hence the conclusion of $(1)$.

(2) Suppose that $\varphi_{\psi, \eta}:(X, \mathcal{S}) \rightarrow(Y, \mathcal{T})$ is continuous. To show that $\varphi_{\psi, \eta}:\left(X, \gamma^{\mathcal{S}}\right) \rightarrow\left(Y, \gamma^{\mathcal{T}}\right)$ is continuous, we have to show that for each $a \in I, k \in K$ and $g \in \gamma_{\eta(k)}^{\mathcal{T}}(a)$, we have $\varphi_{\psi}^{-1}(g) \in \gamma_{k}^{\mathcal{S}}(a)$.

From $g \in \gamma_{\eta(k)}^{\mathcal{T}}(a)=\bigcup_{b>a} \gamma_{\eta(k)}^{\mathcal{T}}(b)$, we know that $g$ has the form of $g=\bigsqcup_{j \in J} \sqcap_{i \in M_{j}} g_{j_{i}}$, where $M_{j}$ 
is a finite index set and $g_{j_{i}} \in \gamma_{\eta(k)}^{\mathcal{T}}\left(b_{j_{i}}\right)\left(b_{j_{i}}>\right.$ a). By Lemma 4.1, $\mathcal{T}_{\eta(k)}\left(g_{j_{i}}\right) \geq b_{j_{i}}>a$. Thus $\mathcal{S}_{k}\left(\varphi_{\psi}^{-1}\left(g_{j_{i}}\right)\right) \geq \mathcal{T}_{\eta(k)}\left(g_{j_{i}}\right)>a$. Moreover, $\varphi_{\psi}^{-1}\left(g_{j_{i}}\right) \in \gamma_{k}^{\mathcal{S}}(a)$. Therefore, $\varphi_{\psi}^{-1}(g)=$ $\bigsqcup_{j \in J} \sqcap_{i \in M_{j}} \varphi_{\psi}^{-1}\left(g_{j_{i}}\right) \in \gamma_{k}^{\mathcal{S}}(a)$.

By the above proposition, we have a functor from the category FSTOP of fuzzy $(E, K)$-soft topological spaces to ASTOP, which is injective on objects.

Given a compatible antichain $(E, K)$-soft $I$ topology $\gamma$ on $X$, then we can construct a fuzzy $(E, K)$-soft topology $\mathcal{T}^{\gamma}$ on $X$ such that for each $f \in\left(I^{X}\right)^{E}$ and $k \in K$,

$$
\mathcal{T}_{k}^{\gamma}(f)=\sup \left\{a \in I_{1} \mid f \in \gamma_{k}(a)\right\} .
$$

We have:

Proposition 4.2 $\mathcal{T}^{\gamma}$ is a fuzzy $(E, K)$-soft topology on $X$, called induced fuzzy $(E, K)$-soft topology by $\gamma$.

Proof. We need to check the axioms (O1)-(O3) of Definition 3.1.

(O1): It is clear that $\mathcal{T}_{k}^{\gamma}\left(\widetilde{E}_{X}\right)=1$ and $\mathcal{T}_{k}^{\gamma}\left(\Phi_{X}\right)=$ 1 , for each $k \in K$.

(O2): Let $\mu$ be any number in $I_{1}$ and $k \in K$ such that $\mu<\mathcal{T}_{k}^{\gamma}(f) \wedge \mathcal{T}_{k}^{\gamma}(g)$. Hence $\mu<\mathcal{T}_{k}^{\gamma}(f)$ and $\mu<\mathcal{T}_{k}^{\gamma}(g)$. Taking a $\lambda \in I$ such that $\mu<$ $\lambda<\mathcal{T}_{k}^{\gamma}(f)$ and $\mu<\lambda<\mathcal{T}_{k}^{\gamma}(g)$, there exists $a_{f}$ and $a_{g}$ such that $\lambda<a_{f} \wedge a_{g}$ and both $f \in \gamma_{k}\left(a_{f}\right)$ and $g \in \gamma_{k}\left(a_{g}\right)$. Then $f \in \gamma_{k}(\lambda)$ and $g \in \gamma_{k}(\lambda)$, moreover $f \sqcap g \in \gamma_{k}(\lambda)$ since $\gamma_{k}(\lambda)$ is an $(E, K)$-soft $I$-topology. That is, $\mathcal{T}_{k}^{\gamma}(f \sqcap g) \geq \lambda>\mu$. Since $\mu$ is arbitrary, we obtain $\mathcal{T}_{k}^{\gamma}(f \sqcap g) \geq \mathcal{T}_{k}^{\gamma}(f) \wedge \mathcal{T}_{k}^{\gamma}(g)$, for each $k \in K$.

(O3): Let $\mu$ be any number in $I_{1}$ and a family of $\left\{f_{j} \mid j \in J\right\} \subseteq\left(I^{X}\right)^{E}$ such that $\mu<\bigwedge_{j \in J} \mathcal{T}_{k}^{\gamma}\left(f_{j}\right)$. Thus $\mathcal{T}_{k}^{\gamma}\left(f_{j}\right)>\mu$ for every $j \in J$. By the definition of $\mathcal{T}_{k}^{\gamma}$, there exists $a_{j}>\mu$ such that $f_{j} \in \gamma_{k}\left(a_{j}\right)$, hence we obtain $\sqcup_{j \in J} f_{j} \in \bigcup_{\beta>\mu} \gamma_{k}(\beta)=\gamma_{k}(\mu)$. This means that $\mu \leq \mathcal{T}_{k}^{\gamma}\left(\sqcup_{j \in J} f_{j}\right)$. Therefore, since $\mu$ is arbitrary, $\mathcal{T}_{k}^{\gamma}\left(\sqcup_{j \in J} f_{j}\right) \geq \bigwedge_{j \in J} \mathcal{T}_{k}^{\gamma}\left(f_{j}\right)$ is obtained.

Proposition 4.3 (1) If $(X, \gamma)$ and $(X, \xi)$ are two objects in ASTOP and they determine the same fuzzy $(E, K)$-soft topology on $X$, then they are equal.

(2) Let $(X, \gamma)$ and $(Y, \xi)$ be $\left(E_{1}, K_{1}\right)$-soft $I$ topological space and $\left(E_{2}, K_{2}\right)$-soft $I$-topological space, respectively. If $\varphi_{\psi, \eta}:(X, \gamma) \rightarrow(Y, \xi)$ is continuous, then $\varphi_{\psi, \eta}$ is continuous with respect to the induced fuzzy soft topologies.

Proof. (1) Let $\tau$ be the same fuzzy $(E, K)$-soft topology induced by $\gamma$ and $\xi$. We want to show $\gamma=$ $\xi$, i.e, $\gamma_{k}(a)=\xi_{k}(a)$ for every $k \in K$ and $a \in I_{1}$. As an alternative way, for each $a \in I_{1}$, we show that for all $b>a, f \in \gamma_{k}(b)$ means $f \in \xi_{k}(a)$. Thus, $\gamma_{k}(a)=$ $\bigcup_{b>a} \gamma_{k}(b) \subseteq \xi_{k}(a)$ and conversely in a similar way. In fact, $f \in \gamma_{k}(b)$ implies $\tau_{k}(f) \geq b>a$. Hence, there exists $c>a$ such that $f \in \xi_{k}(c)$, i.e., $f \in \xi_{k}(a)$.
(2) Suppose that $\varphi_{\psi, \eta}:(X, \gamma) \rightarrow(Y, \xi)$ is continuous. We hope that $\tau_{k}^{\gamma}\left(\varphi_{\psi}^{-1}(f)\right) \geq \tau_{\eta(k)}^{\xi}(f)$ holds for every $k \in K_{1}$ and $f \in\left(I^{Y}\right)^{E_{2}}$.

Taking any number $a \in I_{1}$ such that $a<\tau_{\eta(k)}^{\xi}(f)$, then there exists $b \in I_{1}$ such that $a<b$ and $f \in \xi_{\eta(k)}(b)$. Thus, $\varphi_{\psi}^{-1}(f) \in \gamma_{k}(b)$ since $\varphi_{\psi, \eta}$ : $(X, \gamma(b)) \rightarrow(Y, \xi(b))$ is continuous. We obtain, $\tau_{k}^{\gamma}\left(\varphi_{\psi}^{-1}(f)\right) \geq b>a$. Therefore, since $a$ is arbitrary, we get the conclusion, $\tau_{k}^{\gamma}\left(\varphi_{\psi}^{-1}(f)\right) \geq \tau_{\eta(k)}^{\xi}(f)$.

By above propositions, we obtain the following theorem.

Theorem 4.1 The category FSTOP is isomorphic to the cartegory ASTOP. Hence ASTOP is a topological category over $\mathbf{S E T}^{\mathbf{3}}$.

\section{Conclusion}

In this paper, we gave a characterization of the category FSTOP[5]. In this manner, we constructed ASTOP, the category of antichain soft topological spaces and their continuous mappings. Also, we showed that FSTOP is isomorphic to ASTOP and so, ASTOP is a topological category over $\mathbf{S E T}^{\mathbf{3}}$. From [1], we learn that a topological category inherits all limits and colimits, whenever they exist, from the underlying ground category. Hence, ASTOP has all limits and colimits, since SET has them. For further research, one can try to extend these results to the case of $L$-fuzzy soft topological spaces where $L$ is a completely distributive lattice.

\section{References}

[1] J. Adámek, H. Herrlich, G. E. Strecker, Abstract and Concrete Categories, Wiley, New York, 1990.

[2] B. Ahmad, A. Kharal, On fuzzy soft sets, Advances in Fuzzy Systems, Volume 2009, Article ID 586507.

[3] H. Aktaş, N. Çağman, Soft sets and soft groups, Information Sciences 177 (13):2726-2735, 2007.

[4] A. Aygünoğlu, H. Aygün, Introduction to fuzzy soft groups, Computers and Mathematics with Applications 58:1279-1286, 2009.

[5] A. Aygünoğlu, V. Çetkin and H. Aygün, An introduction to fuzzy soft topological spaces, Hacettepe Journal of Mathematics and Statistics, to be published.

[6] C. L. Chang, Fuzzy topological spaces, Journal of Mathematical Analysis and Applications 24: 182-190, 1968.

[7] N. Çağman, S. Enginoğlu and F. Çitak, Fuzy soft set theory and its applications, Iranian Journal of Fuzzy Systems 8 (3): 137-147, 2011.

[8] Y. B. Jun, Soft BCK/BCI algebras, Computers and Mathematics with Applications 56 (5):14081413, 2008.

[9] A. Kharal, B. Ahmad, Mappings on fuzzy soft classes, Advances in Fuzzy Systems, Volume 2009, Article ID 407890. 
[10] G. J. Klir, B. Yuan, Fuzzy sets and fuzzy logic, Theory and Applications, Prentice-Hall Inc., New Jersey, 1995.

[11] P. K. Maji, R. Biswas, A. R. Roy, Fuzzy soft sets, Journal of Fuzzy Mathematics, 9(3):589602, 2001.

[12] P. K. Maji, A. R. Roy and R. Biswas, An application of soft sets in a decision making problem, Computers and Mathematics with Applications 44 (8-9):1077-1083, 2002.

[13] D. Molodtsov, Soft set theory-First results, Computers and Mathematics with Appl. 37 (4/5):19-31, 1999.

[14] D. Pei, D. Miao, From soft sets to information systems, Granular Computing, 2005 IEEE International Conference on (2), pages 617-621, 2005.

[15] A. R. Roy, P. K. Maji, A fuzzy soft set theoretic approach to decision making problems, Journal of Computational and Applied Mathematics, 203:412-418, 2007.

[16] A. P. Šstak, On a fuzzy topological structure, Suppl. Rend. Circ. Matem. Palermo, Ser II 11:89-103, 1985.

[17] L. A. Zadeh, Fuzzy sets, Information and Control 8: 338-353, 1965.

E-mails: ${ }^{1}$ vildan.cetkin@kocaeli.edu.tr

2 halis@kocaeli.edu.tr 